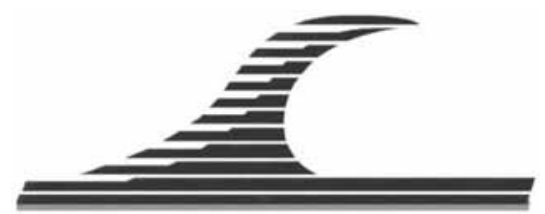

Revue Paralia, Volume 3 (2010) pp 5.1-5.12

Mots-clés : ANEMOC, Mer Méditerranée, Etats de mer, Hindcast, Atlas de houle, Atlas d'états de mer, TOMAWAC

(C) Editions Paralia CFL

\title{
Constitution d'une base de données d'états de mer le long des côtes françaises méditerranéennes par simulations rétrospectives couvrant la période 1979-2008
}

\author{
David MORELLATO ${ }^{1}$, Michel BENOIT ${ }^{2}$
}

1. CETMEF, Technopole Brest-Iroise, BP 5, 29280 Plouzané, France. david.morellato@developpement-durable.gouv.fr

2. Laboratoire d'Hydraulique Saint-Venant, Université Paris Est (laboratoire de recherche commun EDF R\&D, CETMEF, Ecole des Ponts ParisTech), 6 quai Watier, BP 49, 78401 Chatou cedex, France.

michel.benoit@edf.fr

\section{Résumé :}

Une base de données numériques d'états de mer en Méditerranée a été construite à partir de simulations effectuées avec le logiciel TOMAWAC sur la période 1979-2008 afin de compléter la base de données ANEMOC couvrant la façade Atlantique-MancheMer du Nord des côtes françaises. Les champs de vents utilisés en entrée sont issus d'une nouvelle analyse météorologique NCEP2 du U.S. Climate Diagnostics Center de la National Oceanic and Atmospheric Administration (CDC-NOAA). Les résultats du modèle sont comparés à des mesures de bouées et à des mesures satellitaires altimétriques. L'analyse statistique de ces simulations rétrospectives permet d'estimer les conditions moyennes et extrêmes des vagues sur la Mer Méditerranée.

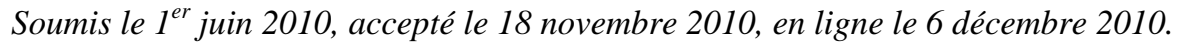

La seule version examinée est celle écrite en français. La ou les autres versions n'étant pas examinées par le comité de rédaction de la revue, sont donc publiées sous l'entière responsabilité du ou des auteurs.

A TRANSLATED VERSION IN ENGLISH IS AVAILABLE ONLINE

Pour citer cet article :

MORELLATO D., BENOIT M. (2010). Constitution d'une base de données d'états de mer le long des côtes françaises méditerranéennes par simulations rétrospectives couvrant la période 1979-2008. Revue Paralia, Vol. 3, pp 5.1-5.12. 


\section{2 : Revue Paralia - Vol. 3 (2010)}

\section{Introduction}

Ces dernières années, le CETMEF et le Laboratoire National d'Hydraulique et Environnement (LNHE) de EDF R\&D ont collaboré pour construire à partir de simulations numériques rétrospectives une base de données continue d'états de mer couvrant le nord-est de l'Océan Atlantique, la Manche et la Mer du Nord avec une résolution plus fine le long des côtes françaises (BENOIT \& LAFON ; 2004, BENOIT et al., 2008). Cette base de données, appelée ANEMOC (pour "Atlas Numérique d'Etats de Mer Océaniques et Côtiers"), couvre une période de temps allant du $1^{\mathrm{er}}$ Janvier 1979 au 31 Août 2002. Une partie des résultats obtenus pendant ce projet sont disponibles sur le site Internet : http://anemoc.cetmef.developpement-durable.gouv.fr. Parmi d'autres applications, cette base de données a été utilisée pour construire des cartes représentant des conditions de vagues moyennes ou extrêmes (LAFON \& BENOIT, 2006).

L’étude présentée suit les mêmes lignes en termes d'objectifs, de méthode et de produits de sortie en se focalisant sur la partie occidentale de la Mer Méditerranée, avec un intérêt particulier sur les côtes Sud de l'Europe (et en particulier France, Espagne et Italie).

\section{Description du modèle numérique utilisé}

\subsection{Le logiciel de modélisation des états de mer TOMAWAC}

Les simulations ont été réalisées avec le modèle de troisième génération spectral TOMAWAC (BENOIT et al. 1996), qui fait partie de la suite hydro-informatique TELEMAC, développé par EDF-LNHE. TOMAWAC résout l'équation d'évolution de la densité spectro-angulaire d'action d'onde (e.g. BRETHERTON \& GARRET, 1969 ; KOMEN et al., 1994) et modélise l'évolution (en espace et en temps) du spectre directionnel des vagues, sous des conditions non stationnaires de forçage par les vents. Il prend en compte l'apport d'énergie par le vent, les interactions non linéaires et les dissipations dues au moutonnement, au frottement sur le fond et au déferlement bathymétrique des vagues en faible profondeur. Un détail intéressant de l'utilisation de TOMAWAC pour des applications côtières est la prise en compte de maillage en éléments finis, de façon à pouvoir raffiner dans les zones de bathymétrie complexe et de trait de côte irrégulier. Le modèle a fait l'objet d'une validation pour la simulation de tempêtes réelles (BENOIT et al., 1996 ; AELBRECHT et al., 1998).

Pour ce projet, une grille numérique a été définie avec une résolution variable sur la Mer Méditerranée (voir section suivante). Le spectre des vagues est décomposé en 21 fréquences en échelle logarithmique avec $\Delta \mathrm{f} / \mathrm{f}=0.122$ (entre $0.04 \mathrm{~Hz}$ et $0.4 \mathrm{~Hz}$ ) et en 36 directions (résolution angulaire constante de 10 degrés). Le pas de sortie des résultats est d'une heure. Le modèle a été utilisé avec un plan d'eau stationnaire (correspondant au niveau moyen de la marée) et sans courant de marée, même si le code peut traiter des courants et des niveaux d'eau non stationnaire. Des simulations couplées avec le modèle 
Constitution d'une base de données d'états de mer le long des côtes françaises méditerranéennes par simulations rétrospectives couvrant la période 1979-2008 : 5.3

hydrodynamique TELEMAC-2D sont à l'étude pour reproduire les interactions houlecourant et feront partie d'une nouvelle phase de ce projet (ANEMOC 2).

\subsection{Le modèle Méditerranée}

Le modèle couvre la Mer Méditerranée $\left(30^{\circ} \mathrm{N}\right.$ à $45^{\circ} \mathrm{N}$ en latitudes et $6^{\circ} \mathrm{W}$ à $36^{\circ} \mathrm{E}$ en longitudes), avec un pas spatial variable, de 2 à $3 \mathrm{~km}$ le long des côtes françaises et de 6 à $10 \mathrm{~km}$ le long des côtes espagnoles et italiennes avec une résolution maximale de $0.5^{\circ}$ sur le reste du domaine. La grille spatiale comprend 8770 nœuds et 16192 triangles (voir figure 1). Aucun spectre n’a été imposé sur les frontières du modèle : toutes les vagues ont été générées à l'intérieur du modèle. Les processus en eau peu profonde (réfraction, shoaling, frottement sur le fond et déferlement) sont pris en compte dans le modèle. Le pas de calcul est de 4 minutes et le temps CPU requis pour modéliser une année complète est de 7 jours sur un PC de bureau.

Les données bathymétriques ont été obtenues à partir d'ETOPO 1 (AMANTE \& EAKINS, 2009, http://www.ngdc.noaa.gov/mgg/global/global.html), et du Service Hydrographique et Océanographique de la Marine (SHOM) avec une haute résolution le long des côtes françaises (figure 1).

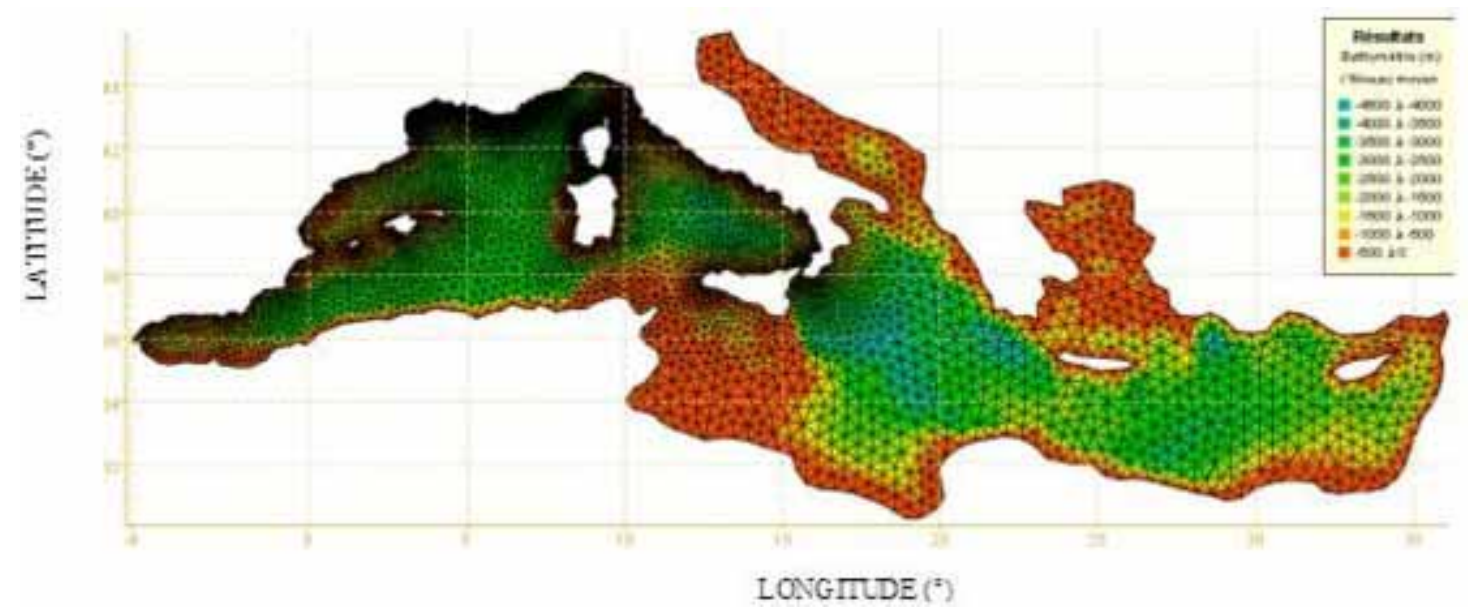

Figure 1. Maillage utilisé pour les simulations avec TOMAWAC.

\subsection{Les champs de vents utilisés}

Le modèle de vagues est forcé par les champs de vents provenant d'une nouvelle analyse NOAA/NCEP (version 2), produite par la NOAA/OAR/ESRL PSD, Boulder, Colorado, USA, disponible sur Internet (http://www.esrl.noaa.gov/psd/, KANAMITSU et al., 2002). Nous utilisons les deux composantes de $U_{10}$ (vitesse moyenne à $10 \mathrm{~m}$ audessus du niveau moyen) produites toutes les 6 heures sur une grille Gaussienne T62 de résolution 1.875 degrés. Les données sont interpolées linéairement en temps et en espace sur la grille de calcul. 
Le modèle est ensuite utilisé pour modéliser les conditions d'états de mer sur 30 ans du 01/01/1979 au 31/12/2008.

\section{Le calage du modèle et la comparaison avec les mesures}

Pour calibrer le modèle numérique, nous avons utilisé la hauteur significative des vagues $\mathrm{H}_{\text {mo }}$ mesurée par radar altimétrique (QUEFFEULOU \& CROIZE-FILLON, 2009) de 1992 à 2008. Les trajectoires des satellites utilisées pour le calage (ERS1, ERS2, ENVISAT, TOPEX, POSEIDON, JASON et GFO) sur la Mer Méditerranée sont représentées à la figure 2.
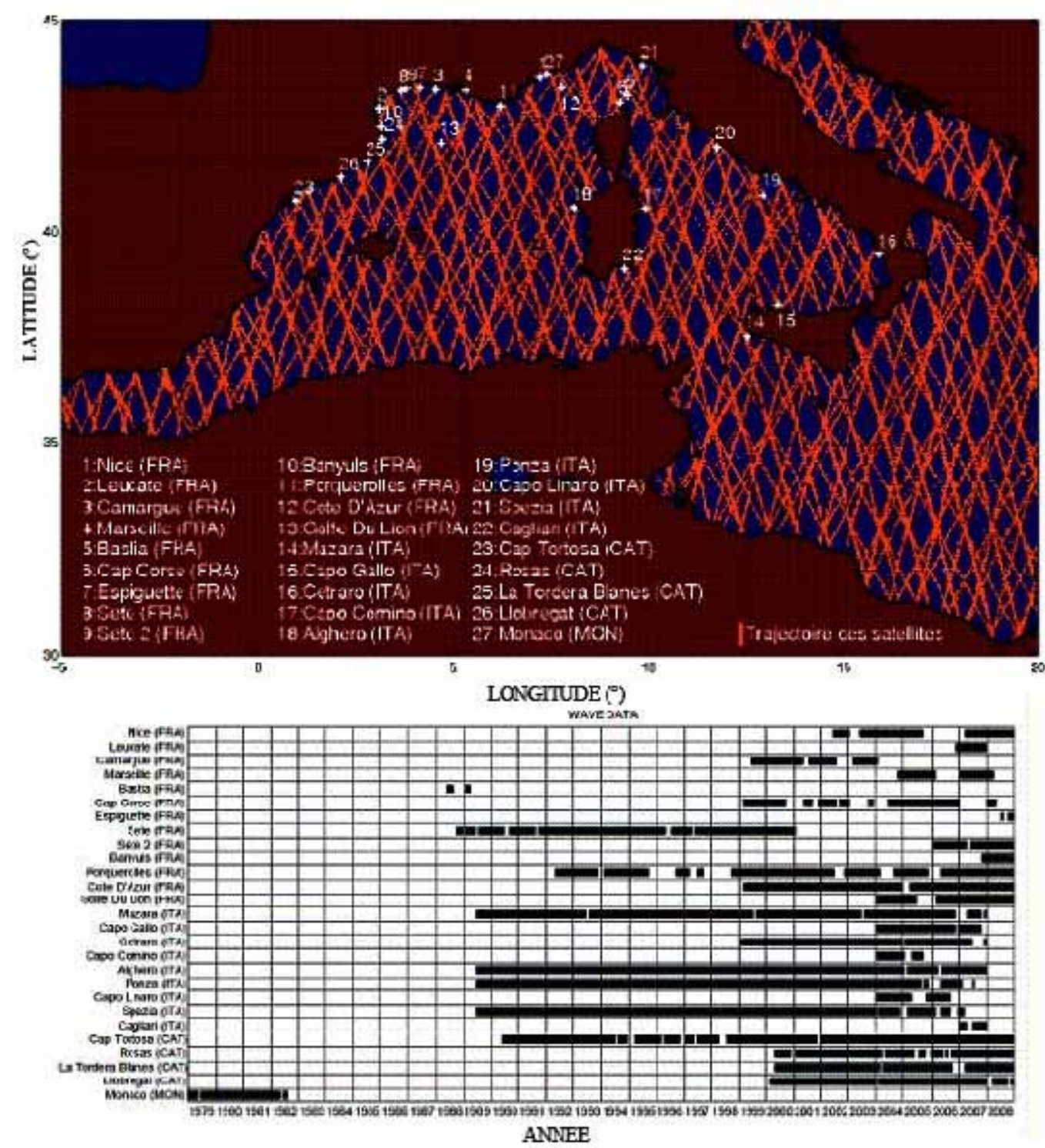

Figure 2. En haut : Positions des bouées et trajectoires des satellites ; En bas : Périodes de mesures des bouées utilisées pour la comparaison. 
Constitution d'une base de données d'états de mer le long des côtes françaises méditerranéennes par simulations rétrospectives couvrant la période 1979-2008 : 5.5

Quand le modèle de vagues a été utilisé avec ses paramètres par défaut et les données de vents originales provenant d'une nouvelle analyse NCEP-2, nous avons obtenu une sous-estimation moyenne $\mathrm{H}_{\text {mo }}$ de 0 à $20 \%$ le long des côtes italiennes et espagnoles et de 0 à $40 \%$ le long des côtes françaises. L'analyse des comparaisons entre les résultats du modèle et les mesures altimétriques révèle que la différence varie avec la position $(\mathrm{x}, \mathrm{y})$ sur la Mer Méditerranée et avec la direction du vent. Ainsi, nous avons déterminé un coefficient de correction appliqué à la vitesse du vent dépendant de la position (x,y) et de la direction du vent pour corriger les biais moyens. La justification de ce type de correction est amenée par le fait que pour certaines prévisions de la houle, la vitesse du vent est mal estimée par le modèle atmosphérique à cause de la présence des montagnes et des irrégularités côtières. Au final, nous avons obtenu deux chroniques temporelles des états de mer nommées "original wind" et "modified wind". Ces chroniques sont comparées aux mesures de 27 houlographes de la Mer Méditerranée occidentale (voir figure 2 pour les périodes de mesures et les positions de ces bouées). Onze de ces bouées proviennent de la base de données CANDHIS du CETMEF (http://candhis.cetmef.developpement-durable.gouv.fr/), deux de METEO-FRANCE, neuf du réseau italien RON (Rete ondametrica Nazionale, http://www.envirtech.org/ron.htm), quatre du réseau catalan XIOM (Xarxa d'Instrumentacion Oceanografica I Meteorologica, http://www.boiescat.org) et une de Monaco.

La comparaison des chroniques mesurées et calculées des paramètres d'états de mer (la hauteur significative des vagues $\mathrm{H}_{\mathrm{mo}}$, la période moyenne $\mathrm{T}_{\mathrm{m} 02}$, la direction moyenne, etc.) montre que les résultats sont clairement améliorés en utilisant les champs de vents modifiés (exemple sur la figure 3). Le NRMSE (Normalised Root Mean Square Error ou RMS normalisé) pour toutes les bouées passent de $40 \%$ pour les vents originaux à $30 \%$ pour les vents modifiés. La figure 4 qui représente la distribution entre les mesures et le modèle pour 6 bouées montre la décroissance du NRMSE avec la modification des champs de vents. Dans le même sens, la pente de la régression linéaire passant par l'origine est proche de 1.

L'analyse des diagrammes quantiles-quantiles (voir figure 5), qui représentent la correspondance entre les quantiles mesurés et calculés (de 1\% à 99\%) de la distribution statistique de $\mathrm{H}_{\text {mo }}$ sur la période simulée, montre aussi de meilleurs résultats du modèle avec la modification des champs de vents. 
5.6 : Revue Paralia - Vol. 3 (2010)

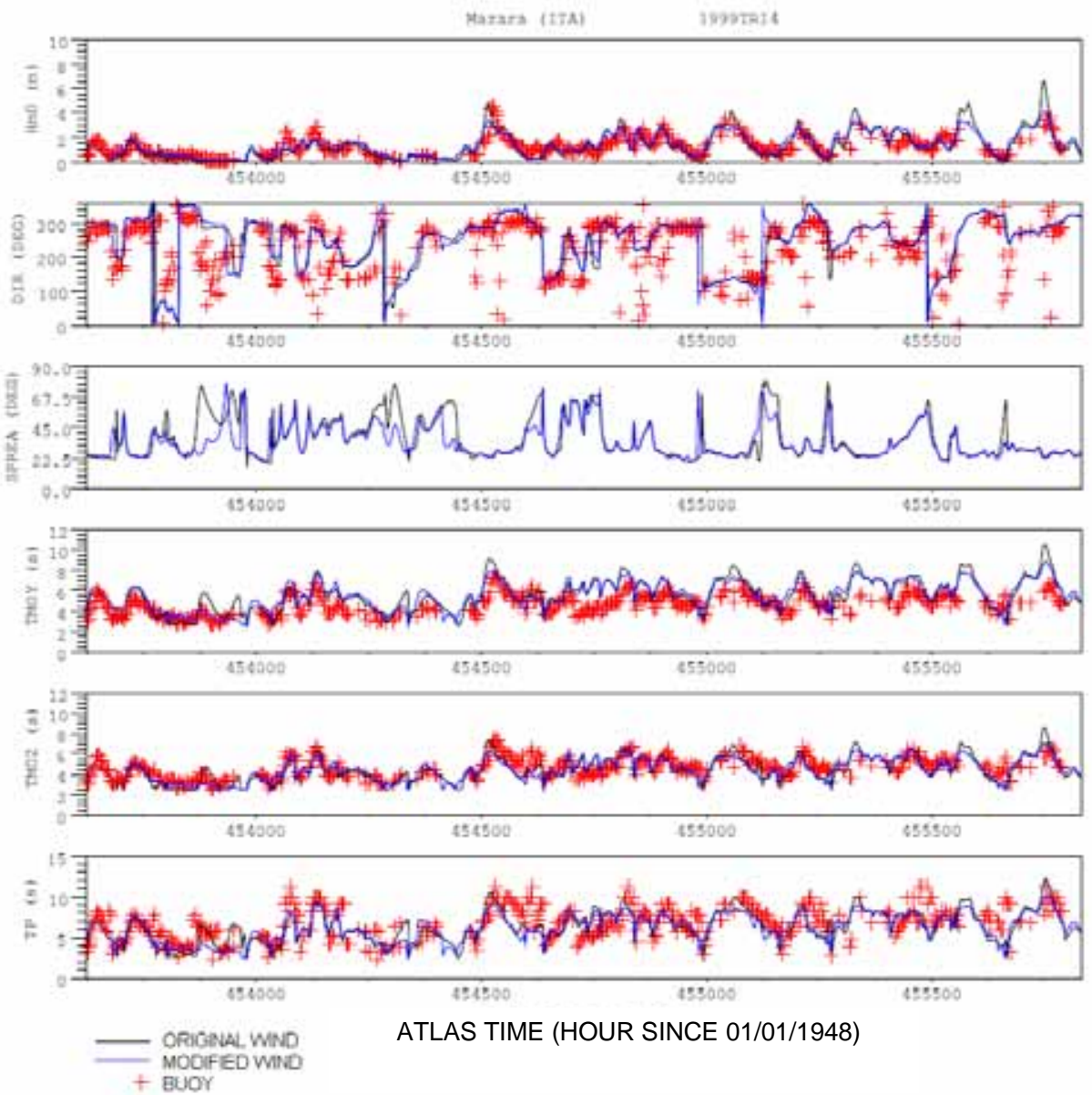

Figure 3. Comparaison des résultats du modèle à la bouée de Mazara pour le dernier trimestre de 1999.
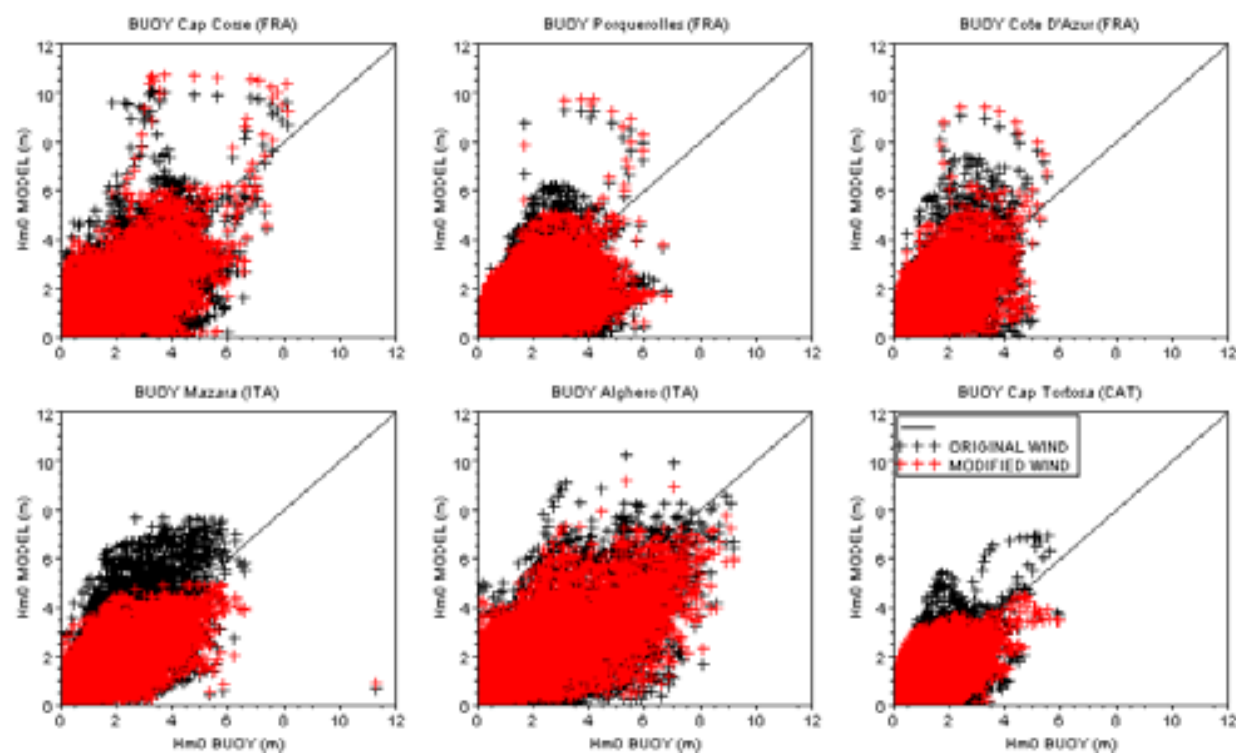

Figure 4. Distribution de $H_{\text {mo }}$ entre mesures et modèle pour 6 bouées. 
Constitution d'une base de données d'états de mer le long des côtes françaises méditerranéennes par simulations rétrospectives couvrant la période 1979-2008 : 5.7
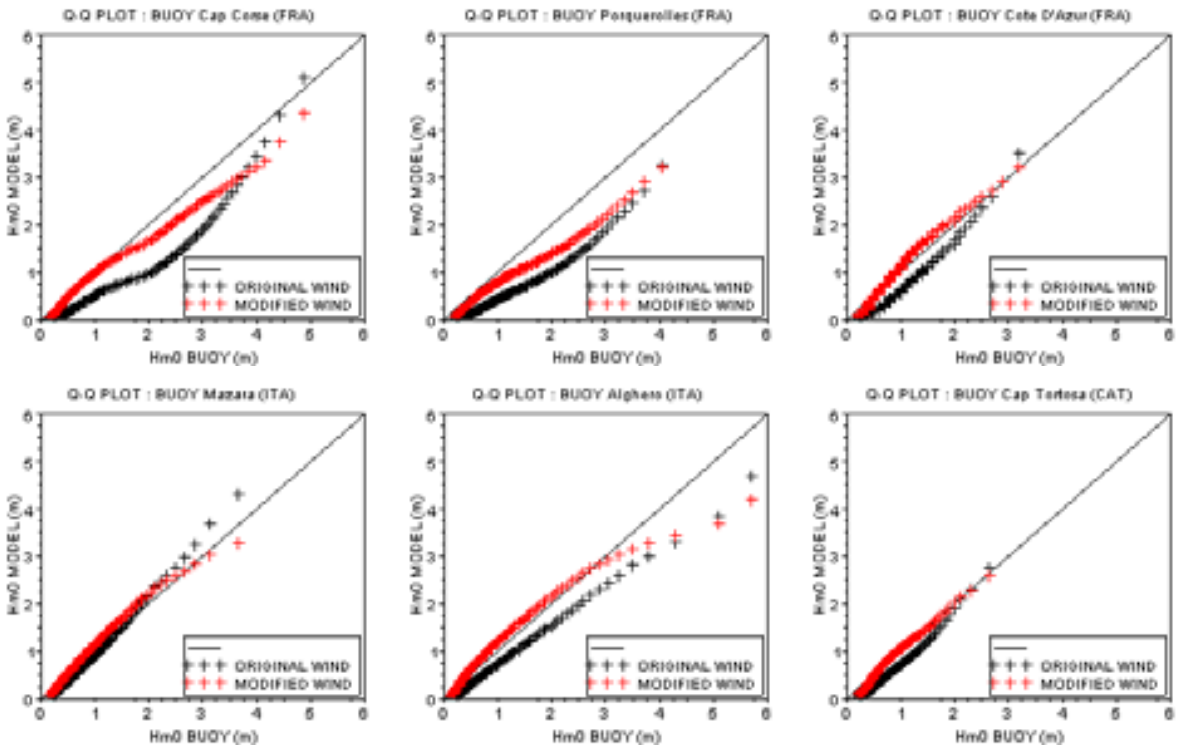

Figure 5. Diagramme Quantile-Quantile (Q1 à Q99) entre mesures et modèle pour 6 bouées.

\section{Quelques résultats globaux}

Une fois la base numérique d'états de mer construite, nous pouvons utiliser les chroniques des paramètres d'états de mer pour produire des diagrammes synthétiques couvrant l'ensemble de la Mer Méditerranée. Quelques exemples sont donnés ici pour la moyenne de la hauteur des vagues significatives (voir figure 6), la moyenne de la puissance de vagues (voir figure 7) et le quantile Q99 de la hauteur significative des vagues (c’est-à-dire la hauteur des vagues qui est dépassée 1\% du temps, voir figure 8).

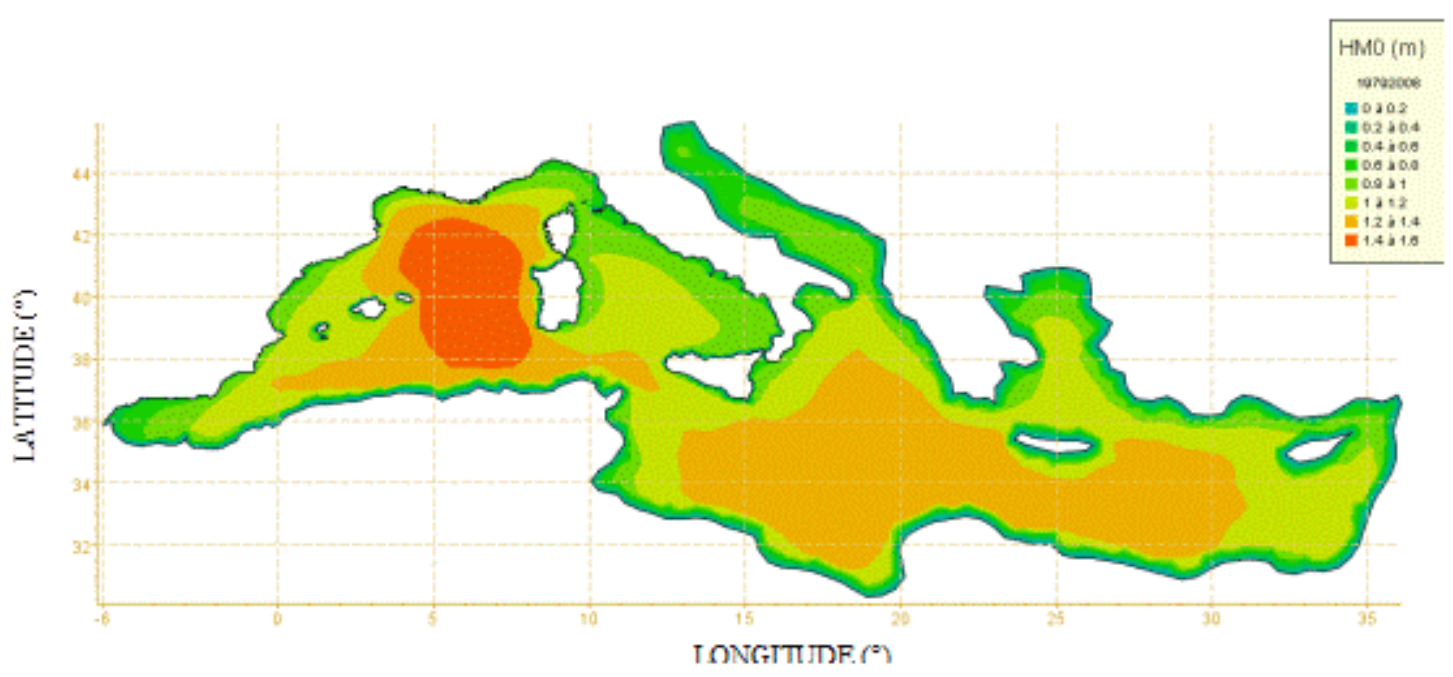

Figure 6. Moyenne des vagues $H_{\text {mo }}$ calculée sur la période 1979-2008. 


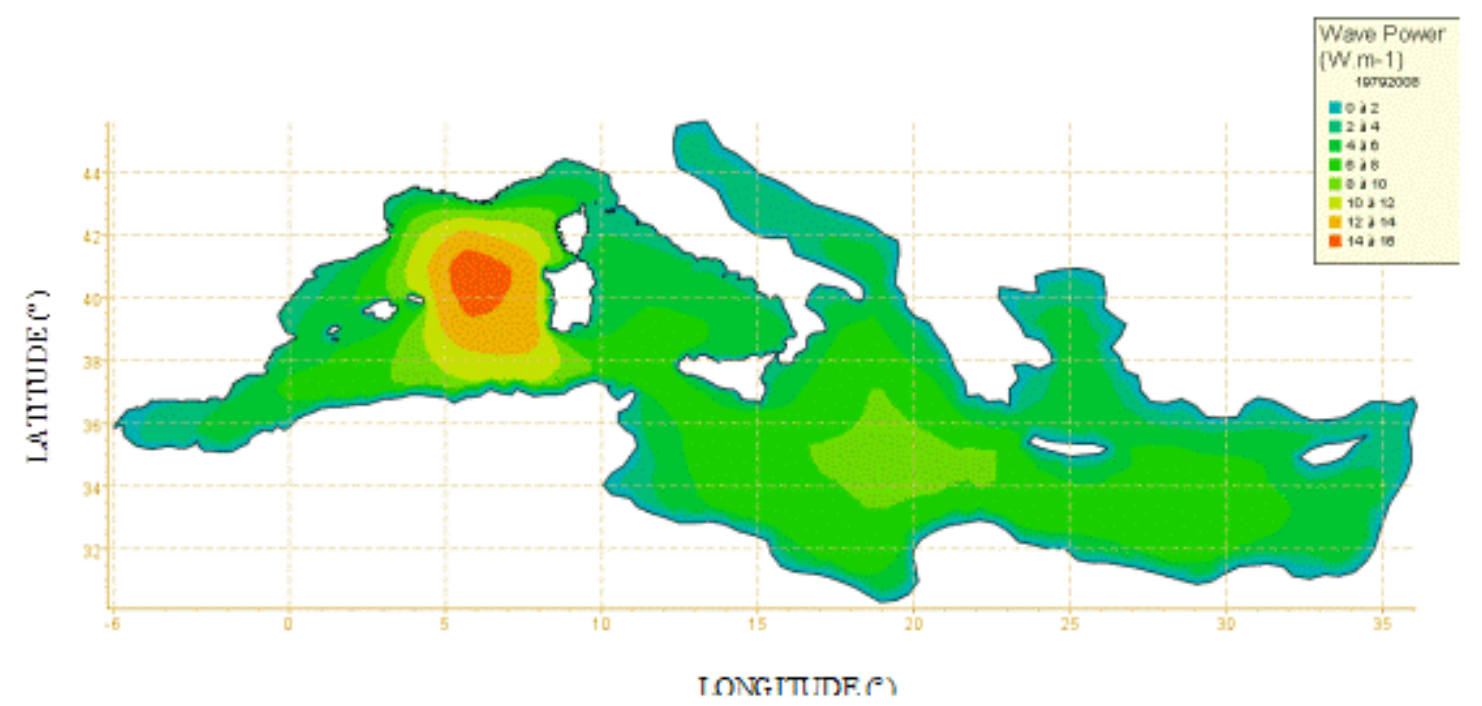

Figure 7. Moyenne de la puissance des vagues calculée sur la période 1979-2008.

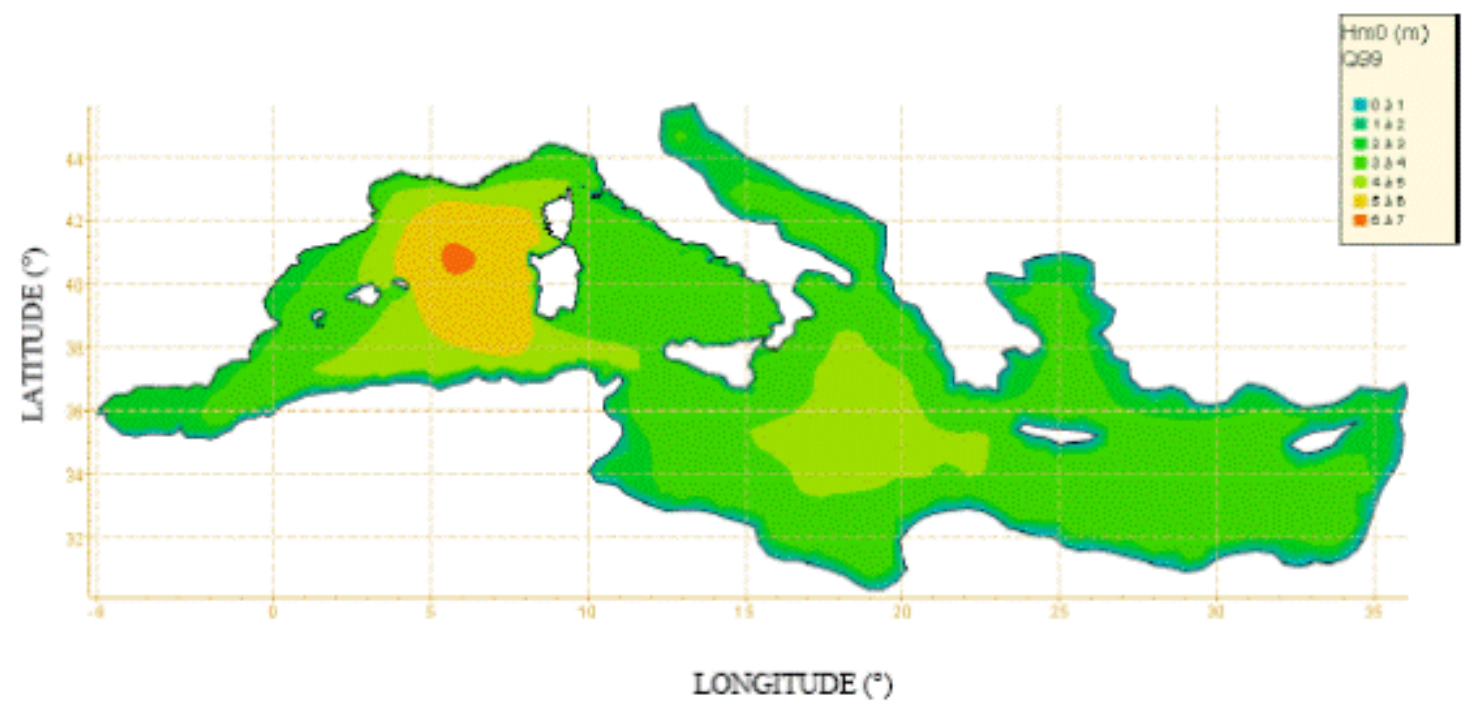

Figure 8. Quantile 99 des vagues calculé sur la période 1979-2008.

\section{Le nouveau site internet ANEMOC}

La base de données ANEMOC sur l'Océan Atlantique, la Manche, la Mer du Nord et la Mer Méditerranée est maintenant complète dans sa première version. Une partie des résultats de ce travail est disponible sur le nouveau site internet ANEMOC où 3 niveaux de données sont identifiés (voir figure 9) :

- Niveau 1 : Chroniques de 6 paramètres d'états de mer sur 750 sites, (gratuit pour des applications de recherche, payant pour des applications commerciales) ;

- Niveau 2 : Analyse des climatologies (annuelles, estivales et hivernales) sur 150 sites (voir figure 10), (gratuit) ;

- Niveau 3 : Résultats de calculs d'extrêmes sur 75 sites, basés sur la méthode POT (Peak-Over Threshold method), (voir figure 11), (gratuit). 
Constitution d'une base de données d'états de mer le long des côtes françaises méditerranéennes par simulations rétrospectives couvrant la période 1979-2008 : 5.9

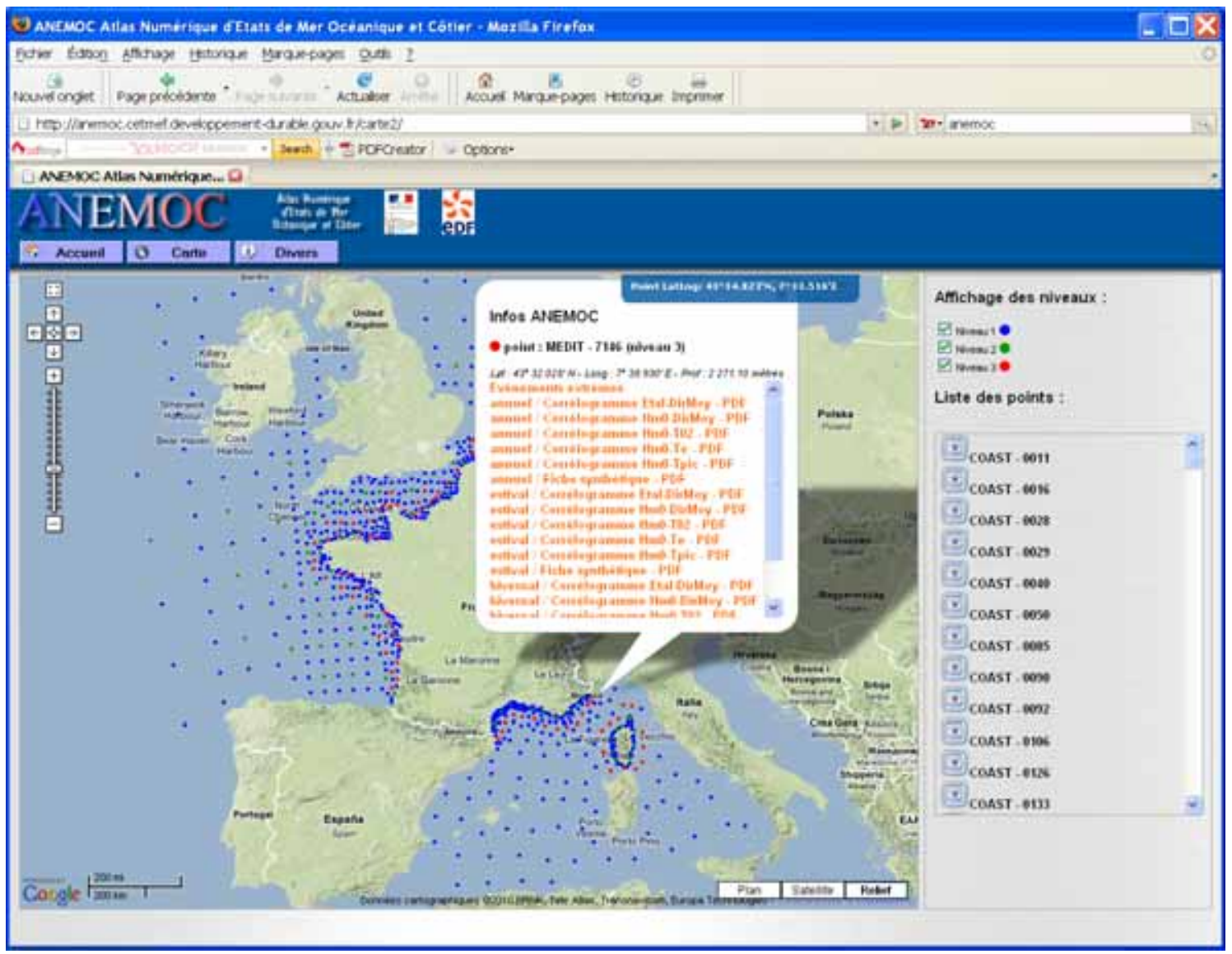

Figure 9. Le site internet ANEMOC.

\section{Perspectives}

Une version ANEMOC 2 est en cours d'élaboration avec le LNHE avec les améliorations suivantes :

- Analyse plus détaillée des composantes d'états de mer,

- Amélioration de la modélisation des processus physiques dans TOMAWAC,

- Extension et validation vers les faibles profondeurs,

- Simulations couplées avec un modèle hydrodynamique pour représenter les interactions avec la marée, les courants et les surcotes,

- Utilisation de champs de vents méso-échelle,

- Assimilation des données altimétriques. 


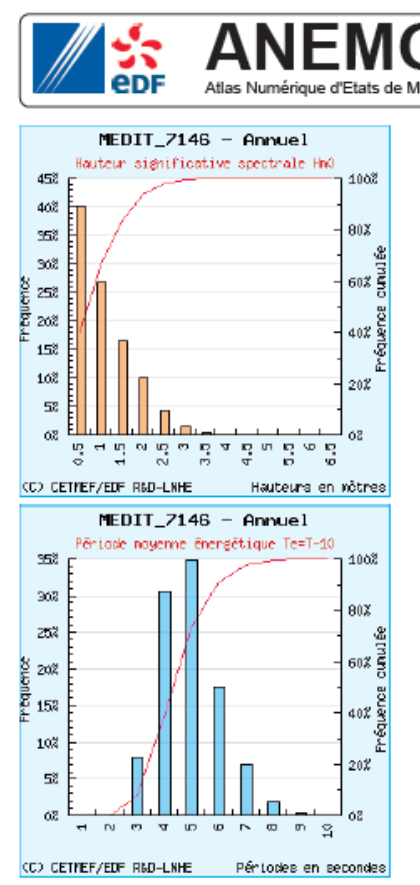

(n)

Point du modèle : MEDIT 7146

Coordonnées: $\quad 7^{\circ} 39.93^{\prime} \mathrm{E} / 43^{\circ} 32.028^{\circ} \mathrm{N}$ Hauteur d'eau dans le modèle : $\quad 2271.10$ mètres
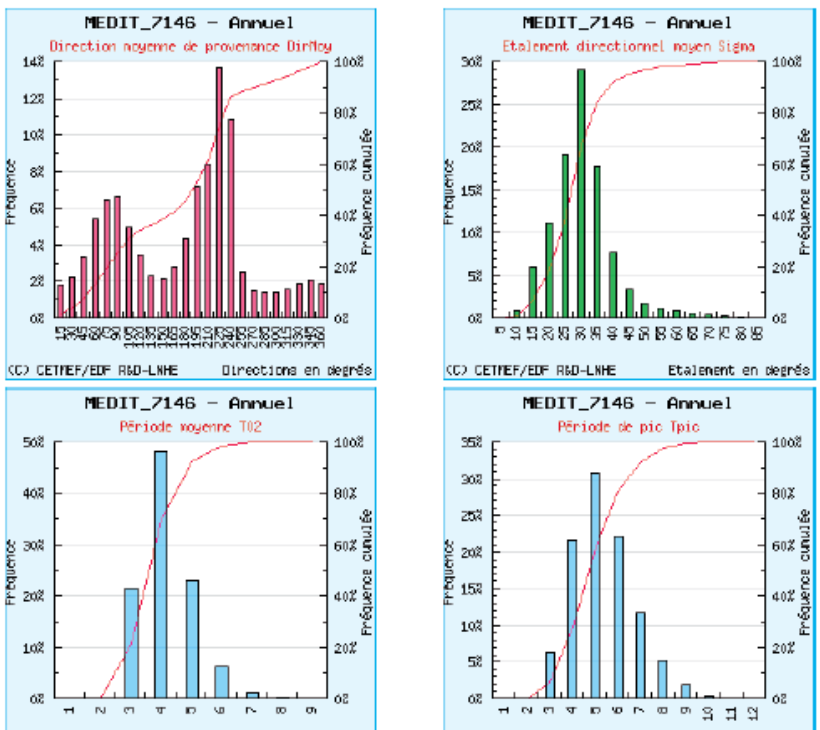

(C) CEITEF/EEF RLD-LNHE Etalenent en deerses

MEDIT_7146 - Anmue

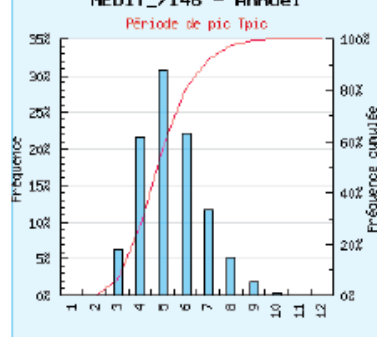

(C) CEITEF/EEF RED-LKHE PÉrLodes en secondes

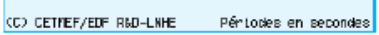

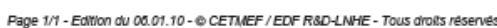

\section{$\because$ ANEMOC \\ Poet du modic MEDIT 7146

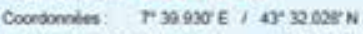

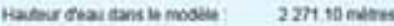

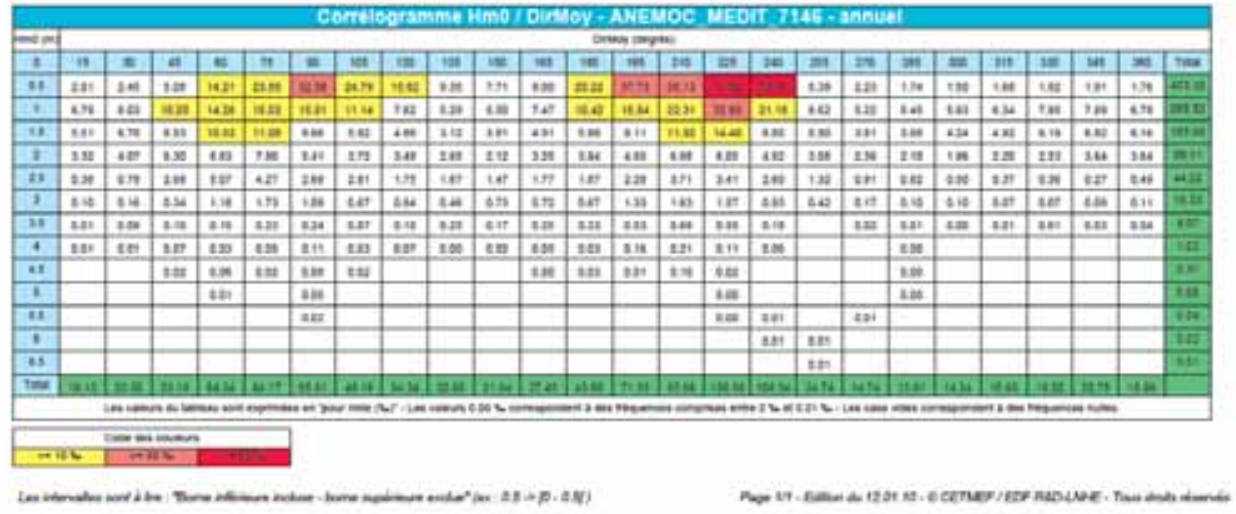

Figure 10. Données de Niveau 2. 
Constitution d'une base de données d'états de mer le long des côtes françaises méditerranéennes par simulations rétrospectives couvrant la période 1979-2008 : 5.11
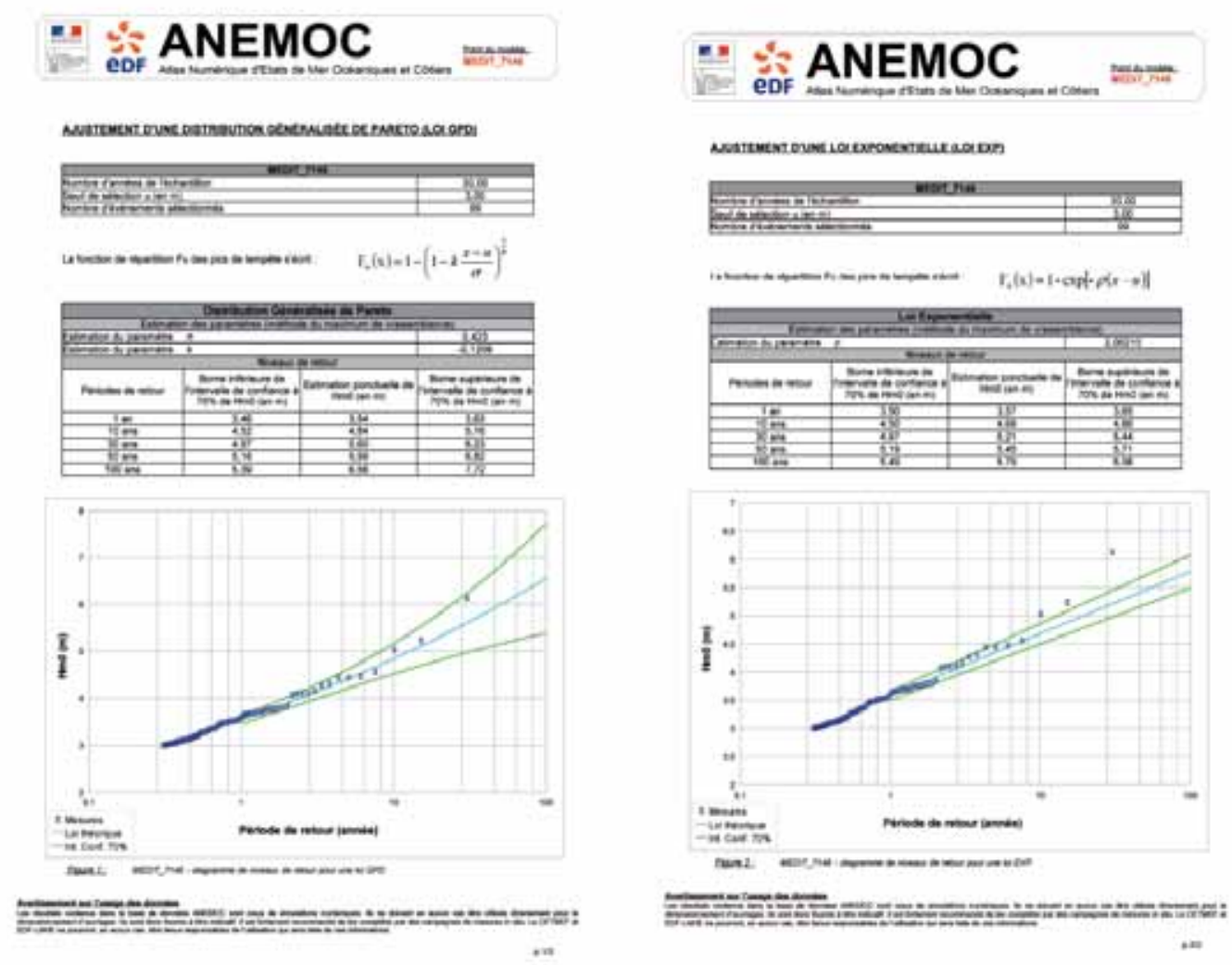

Figure 11. Données de Niveau 3.

\section{Remerciements}

Ce projet est une collaboration entre EDF-LNHE et le CETMEF. Nous remercions Météo-France, la XIOM, le RON, IFREMER, la NOAA et le SHOM pour l'accès à leurs données.

\section{Références bibliographiques}

AELBRECHT D., BENOIT M., MARCOS F., GOASGUEN G. (1998). Prediction of offshore and nearshore storm waves using a third generation spectral wave model, Proc. $8^{\text {th }}$ Int. Offshore and Polar Eng Conf. (ISOPE), vol. III, pp 71-76.

AMANTE C., EAKINS B.W. (2009). ETOPO1 1 Arc-Minute Global Relief Model: Procedures, Data Sources and Analysis. NOAA Technical Memorandum NESDIS NGDC-24, 19 p, March 2009.

BENOIT M., MARCOS F., BECQ F. (1996). Development of a third generation shallow water wave model with unstructured spatial meshing. Proc. $25^{\text {th }}$ Int. Conf. on Coastal Eng. (ICCE’1996), Orlando (Floride, USA), pp 465-478.

BENOIT M., LAFON F. (2004). A nearshore wave atlas along the coasts of France based on the numerical modelling of wave climate over 25 years. Proc. $29^{\text {th }}$ Int. Conf. on Coastal Eng. (ICCE’2004), Lisbonne (Portugal), pp 714-726. 
BENOIT M., LAFON F., GOASGUEN G. (2008). Constitution et exploitation d'une base de données d'états de mer le long des côtes françaises par simulation numérique sur 23 ans. Base ANEMOC en Atlantique - Manche - Mer du Nord. European Journal of Env. and Civil Eng., Vol. 12/1-2, pp 35-50. doi:10.3166/ejece.12.35-50

BRETHERTON F.P., GARRET, C.J.R., (1969). Wavetrains in inhomogenous moving media. Proc. Roy. Soc. London, Series A, 302, pp 529-554.

KANAMITSU M., EBISUZAKI W., WOOLLEN J., YANG S.-K., HNILO J.J., FIORINO M., POTTER G.L. (2002). NCEP-DEO AMIP-II Reanalysis (R-2)1631-1643, Bul. of the Atmos. Met. Soc.

KOMEN G.J., CAVALERI L., DONELAN M., HASSELMANN K., HASSELMANN S., JANSSEN P.A.E.M. (1994). Dynamics and modelling of ocean waves. Cambridge Univ. Press, 532 p. doi:10.1017/CBO9780511628955

LAFON F., BENOIT M. (2006). Estimation of extreme wave conditions from hindcast simulations with application to the wave climate along French coasts. Proc. $30^{\text {th }}$ Int. Conf. on Coastal Eng. (ICCE'2006), San Diego (Californie, USA), pp 739-751.

QUEFFEULOU P., CROIZE-FILLON D. (2009). La mesure satellite de hauteur de vague par altimètre. État des lieux, application à la climatologie et à la modélisation des états de mer. AMA 2009. Les ateliers de modélisation de l'atmosphère, Toulouse, 27-29 janvier 2009. 